
\title{
3 Research Square \\ Effects of Vitrified Cryopreservation Duration on the IVF and Neonatal Outcomes
}

\section{Yuling Mao}

The Third Affiliated Hospital of Guangzhou Medical University

\section{Ping Yin}

The Third Affiliated Hospital of Guangzhou Medical University

\section{Yanfen Luo}

The Second Affiliated Hospital of Guangzhou University of Chinese Medicine

\section{Jingda Qiao}

Guangzhou Medical University Second Affiliated Hospital

\section{Lei Li ( $\nabla$ lileigo@foxmail.com )}

Third Affiliated Hospital of Guangzhou Medical University https://orcid.org/0000-0002-7544-2191

\section{Research Article}

Keywords: Embryo cryopreservation, implantation, live birth, post-thaw survival, storage time

Posted Date: January 4th, 2022

DOI: https://doi.org/10.21203/rs.3.rs-1212906/v1

License: (c) (1) This work is licensed under a Creative Commons Attribution 4.0 International License. Read Full License 


\section{Abstract}

Objective: To evaluate the impact of cryopreservation storage duration on embryo viability, implantation competence, pregnancy outcome and neonatal outcomes.

Design: Retrospective study.

Setting: Center for Reproductive Medicine区The Third Affiliated Hospital of Guangzhou Medical University.

Patient(s): In vitro fertilization patients who had vitrified cryopreserved embryos and following the first frozen embryo transfer cycles from January 2004 to August 2019. A total of 31143 patients met the inclusion criteria and were grouped according to the storage time (20926 patients in Group 1 with storage time $<3$ months, 6472 patients in Group 2 with storage time between 3 and 6 months, 2237 patients in Group 3 with storage time between 6 and 12 months and 746 in Group 4 with storage time between 12 and 24 months, 762 patients in Group 5 with storage time $>24$ months).

Intervention(s): None.

Main Outcome Measure(s): In the total FET cycles, the embryo survival rate was decreased significantly with the increase of cryopreservation time, and the highest rate was $98.63 \%$ in the $1-3$ months group, and the lowest was $71.13 \%$ in the $>=731$ days group $(P<0.01)$. The HCG positive rate $(57.85 \%)$ and clinical pregnancy rate $(55.26 \%)$ in the $1-3$ months group were the highest $(P<0.01)$. The $>=731$ group had the lowest sex ratio of 0.96 . There were no significant differences in neonatal birth weight, neonatal height and congenital anomalies among the groups ( $P>0.05)$.

Result(s): Length of storage time had a significant effect on post-thaw survival and outcomes for IVF cycles.

Conclusion(s): With the prolongation of cryopreservation time, the embryonic survival rate and pregnancy rate were decreased significantly. Short-term cryopreservation ( $<=3$ months) can obtain higher clinical pregnancy rate. Therefore, although long-term hryopreservation of the embryo has no effect on the health of the new baby, but hryopreserved embryos should be recovery as soon as possible if condition allows.

\section{Introduction}

In assisted reproductive technology (ART), cryopreservation of embryos enables excess embryos can be better used. During cryopreservation, there are various kinds of damage risks, including mechanical damage caused by ice crystal formation and permeable injury as cryopreservation agent and cold shock injury due to cooling[1-3].

1985, Rei et al.[4] applied an innovative approach of "vitrification" by using non constant high concentration of cryoprotectant and rapid temperature change to minimize the relationship with ice crystal formation related to damage. Vitrified freezing is defined by making increase viscosity with high 
cooling rate and solution at low temperature without forming ice crystal thus plays a protective role[5]. In recent years along with refrigerant formulation, the improvement and optimization of freezing technology, vitrification cryopreservation technology has been the replacement of slow-speed freezing technique became the most suitable for cryopreservation of cells. Compare that to slow freezing, vitrification reduces ice crystal formation[6, 7] \{Zegers-Hochschild, $2009 \# 1693\}$, improve the embryo freezing survival rate, clinical pregnancy rate and the live birth rate[8, 9].

However, the high concentration of dimethyl sulphoxide (DMSO), a cryoprotective agent used in vitrification reagent, has certain embryonic toxicity[10-12], and whether the embryo samples are contaminated by long-term unprotected exposure to liquid nitrogen has been concerned by people. The purpose of this study was to investigate the effect of vitrification cryopreservation time on the outcome of freezing-thawing embryo transplantation, and to provide a better reference for clinical work.

More than 30000 frozen-thawed embryo transfer (FET) cycles were performed in our reproduction center since the initiation of our vitrification technique. In this study, we explored the impact of storage time on embryo viability, pregnancy outcomes and neonatal outcomes among 31143 patients with the first vitrified embryo transfer cycles following a freeze-all strategy during the period from January 2004 to August 2019.

\section{Materials And Methods}

\section{Study design and participants}

The data was collected from Center for Reproductive Medicine, The Third Affiliated Hospital of Guangzhou Medical University, Guangzhou, China. In vitro fertilization patients who had vitrified cryopreserved embryos and following the first frozen embryo transfer cycles from January 2004 to August 2019.A total of 31143 patients met the inclusion criteria and were grouped according to the storage time (20926 patients in Group 1 with storage time $<3$ months, 6472 patients in Group 2 with storage time between 3 and 6 months, 2237 patients in Group 3 with storage time between 6 and 12 months and 746 in Group 4 with storage time between 12 and 24 months, 762 patients in Group 5 with storage time $>24$ months). The study had been approved by the ethics committee of the Third Affiliated Hospital of Guangzhou Medical University. And written informed consent was obtained from each participant.

\section{ART procedures}

Women were monitored and managed according to the hospital's clinical protocols. Various controlled ovarian stimulation (COS) protocols were used, with 150-450 IU/day of recombinant FSH or human menopausal gonadotropin in a gonadotropin-releasing hormone antagonist protocol, a long agonist protocol, or a short agonist protocol. The protocols were determined according to each patient's characteristics (age, body mass index (BMI), AFC and AMH). Transvaginal oocyte retrieval was scheduled 35-36 $\mathrm{h}$ after HCG injection. ART was performed per standard operating procedure of the hospital. 
Fertilization was assessed by the appearance of two pronuclei. The luteal phase was supported by vaginal administration of micronized progesterone $(400 \mathrm{mg} / \mathrm{d})$ started on the day of ovarian puncture.

\section{Embryo Cryopreservation Techniques and Thawing Protocols}

The embryos were frozen and thawed in accordance with the protocols of the Vitrification Kit (Kato Corporation, Shizuoka, Japan). Initially, the embryos were exposed to an equilibration solution (ES) for 5 min at room temperature(cleavage embryo) or $2 \mathrm{~min}$ at $37^{\circ} \mathrm{C}$ (blastocyst). After that, the embryos were transferred to a vitrification solution (VS) for $45 \mathrm{~s}$. Finally, embryos were set on a cryotop strip (Kato Company, Japan) in a small volume and were plunged into liquid nitrogen as soon as possible. The embryologists have been well trained to perform vitrification technically. The embryos were stored at a constant temperature of $-196^{\circ} \mathrm{C}$ in the liquid phase of liquid nitrogen tank (Taylor Wharton HC35, Theodore, AL, USA). The level of liquid nitrogen is kept under constant surveillance manually by highly skilled embryologists to prevent suboptimal storage conditions, and the liquid nitrogen tank is refilled manually twice a week.

Vitrified embryos were thawed by a rapid thawing method on the morning of embryo transfer. For the thawing process of vitrified embryos, the embryos unloaded from the carriers were immediately submerged into the thawing solution for $1 \mathrm{~min}$ at $37^{\circ} \mathrm{C}$. Then, the embryos were transferred into the diluent thawing solution for $3 \mathrm{~min}$ at room temperature. At the final step, the embryos were moved to the wash solution twice for 3 min at room temperature. After that, the embryos were cultured in culture medium at $37^{\circ} \mathrm{C}$ under the gas phase of $5 \% \mathrm{CO}_{2}$ and $5 \% \mathrm{O}_{2}$ in an incubator until transfer.

\section{Endometrial preparation for FET cycle, and embryo transfer}

Endometrial preparation for FET cycle in this study was achieved by natural cycle (NC) or hormone replace treatment (HRT) programs. The ovulation in NC program was determined by monitoring follicular development with transvaginal ultrasonography and hormone levels. The patients in HRT-FET cycles were treated with daily oral estradiol valerate tablets (Progynova, Bayer, Germany) since the second to fourth day of menstruation. When the endometrial thickness reached $7 \mathrm{~mm}$ or thicker, $40 \mathrm{mg} /$ day progesterone was intramuscularly administered daily. One or two thawed embryos were transfer on the fourth (cleavage-stage embryo) or sixth day (blastocyst) after ovulation or progesterone injection using a softtipped Wallace (PortexLed., Hythe, United Kingdom) catheter under ultrasound guidance. All patients received luteal support with progesterone after embryo transfer. If transvaginal ultrasound showed gestational sac and embryonic heartbeat 4-6 weeks after embryo transfer, luteal support was continued until 10 weeks of gestational age.

\section{Outcome measure}

The reproductive outcome of this study included embryo viability, implantation competence, pregnancy outcome. Neonatal outcomes included sex ratio, birth weight, birth height and congenital anomalies. Live birth was defined as the delivery of any viable neonate who was 28 weeks of gestation or older. Clinical 
pregnancy was defined as the present of gestational sac on ultrasound at 6-8 weeks of gestation; low birth weight was defined as the birth weight less than $2500 \mathrm{~g}$ and high birth weight more than $4000 \mathrm{~g}$.

\section{Statistical analysis}

The statistical analysis was performed using the Statistical Package for Social Science (SPSS) version 22.0. Descriptive data are presented as mean with 1 SD. The differences between groups were tested using the ANOVA test for continuous variables and the Pearson's chisquare test for categorical variables. Multivariable logistic regression was performed to explore the effect of storage time on pregnancy outcome or neonatal outcome after controlling for potential confounders, including maternal age and embryo quality. $P<0.05$ was considered statistically significant.

\section{Results}

A total of 31143 first FET cycles after a freeze-all strategy met study inclusion criteria and were included into the analysis. All FET cycles were divided into five groups according to the storage time (20926 patients in Group 1 with storage time $<3$ months, 6472 patients in Group 2 with storage time between 3 and 6 months, 2237 patients in Group 3 with storage time between 6 and 12 months and 746 in Group 4 with storage time between 12 and 24 months, 762 patients in Group 5 with storage time $>24$ months).

The IVF patients' demographics and cycle characteristics for all embryos are presented in Table 1. The age range of IVF patients at the time of embryo freezing was 27-40 years. Embryo storage time ranged from 30 to 1660 days for all embryos. Maternal age at embryo transfer increased with longer storage time $(P<0.001)$. No difference was seen in type of infertility, infertility causes and number of embryos transferred across all storage time groups. More patients underwent blastocyst transfer in Group 1-2 than in Group 3-5.

Comparisons of FET pregnancy outcomes between the five groups stratified by storage time were summarized in Table 2. In the total FET cycles, the embryo survival rate was decreased significantly with the increase of cryopreservation time, and the highest rate was $98.63 \%$ in the $1-3$ months group, and the lowest was $71.13 \%$ in the $>=731$ days group $(P<0.01)$. The HCG positive rate $(57.85 \%)$ and clinical pregnancy rate $(55.26 \%)$ in the $1-3$ months group were the highest $(P<0.01)$. The $>=731$ group had the lowest sex ratio of 0.96 . Analysis of the Group1 data had the best outcomes: implantation rate of $42.28 \%$, clinical pregnancy rate per transfer of $55.26 \%$, live birth rate per transfer of $19.01 \%$, and multiplets rate of $30.52 \%$.

Comparisons of neonatal outcomes between the five groups stratified by storage time were presented in Table 3. When FET cycles following the different storage time, no significant differences were found for the mean birth weight, birth height and rates of congenital anomalies. The sex ratios (male/female) were greater than 1 in groups 1,3 and 4, while the proportion of girls was greater than that of boys in groups 2 and 5. There are a total of 178 infants presented defects in the circulatory system, nervous system, 
digestive system (congenital esophageal atresia), circulation system (hemangioma), musculoskeletal system, external ear, cleft lip and palate.

Multivariable logistic regression was performed to explore the effect of storage time on pregnancy outcome or neonatal outcome after controlling for potential confounders, including maternal age and embryo quality. The result is the same as before.

\section{Discussion}

The results of this study show that with the extension of freezing time, embryos survival rate, the chance of positive HCG, clinical pregnancy significantly reduced. Earlier studies suggested that after long-term storage, the survival rate and the pregnancy rate of embryos is a trend of decrease after 6-15 months[13]. Testart et al.[14]found increased rates of human embryonic cell death with cryo-storage of only several months. But there are also studies that have found that there was no significant difference in the influence of low temperature preservation time on the quality, survival, implantation capacity and live potential of resuscitated embryos $[15,16]$. Cohen et al. [17]demonstrated no deleterious effects from cryostorage. Animal studies and theoretical modeling speculate that a frozen mammalian embryo should not be influenced by storage time for several thousand years[18]. Surprisingly, few clinical data are available to address this clinically relevant question.

Embryo cryopreservation technology has developed from slow freezing to routine use vitrified cryopreservation[19-21], evaluated carefully for its potential impact on IVF and embryos and perinatal outcomes are significant. Kuang et al. [22]analyzed the effect of freezing time on pregnancy and neonatal outcomes within 2 years, but did not discuss the condition of embryos frozen for longer than 2 years. This study examined 31143 cycles of FET which storage time ranged from 30 to 1660 days in our center. Our results are similar to kuang's, for example, the embryo implantation rate and pregnancy rate decreased gradually with the extension of freezing time. However, there were also some inconsistences, for example, they thought that the freezing time of embryos would not affect the thawing survival rate, but our data showed that the thawing survival rate of embryos would decrease with the extension of freezing time, especially for the group of embryos frozen for more than 2 years, the thawing survival rate of embryos was only $71 \%$. During vitrification, embryos are exposed to cold cryoprotectant and directly bonded with liquid nitrogen in an open vitrification system. Long time exposure of one or more links to touch may alter the early embryo development and influence its survival potential.

The age of the woman and the type of embryo transferred may affect the pregnancy outcome. As our data showed, group 1 had the youngest female and the highest proportion of transplanted blastocysts, so the good pregnancy outcome of group 1 should be excluded from these interfering factors. Multivariable logistic regression was performed to explore the effect of storage time on pregnancy outcome outcome after controlling for potential confounders. The results showed that with the extension of freezing time, the pregnancy outcome still decreased. These results show that the poor pregnancy outcome is indeed caused by the long freezing time, not because of the woman's age and blastocyst. 
There was no statistical difference in neonatal outcomes such as birth weight and height. While, in all cases, the sex ratio was higher in 1,3 and 4 group, with statistical difference $(P<0.05)$. It is known that younger women and blastocysts significantly increased the proportion of male babies. So multivariable logistic regression was performed to explore the effect of storage time on neonatal outcome after controlling for these confounders. which further indicated that the difference in sex ratio was caused by storage time.

\section{Conclusions}

In summary, this study considers that with the extension of cryopreservation time, embryo resuscitation rate will decrease. Short-term storage (no more than 3 Months) can obtain a higher clinical pregnancy rate. Therefore, although long-term hryopreservation of the embryo has no effect on the health of the new baby, but hryopreserved embryos should be recovery as soon as possible if condition allows. However, for patients who cannot be thawed and transplanted in a short period of time for various reasons, they can also choose long-term frozen embryos with a certain success rate. There was a successful pregnancy case in our center that transplanted embryos frozen for 10 years.

\section{Declarations}

\section{Acknowledgements}

Not applicable.

\section{Authors'contributions}

YLM designed research. YLM, PY and YFL analyzed data. JDQ wrote the manuscript. LL revised the manuscript. YLM contributed to the review and revision of manuscript. All authors read and approved the final manuscript.

\section{Funding}

This study was supported by the National Natural Science Foundation of China (No., 81871211 to Lei Li) and (No. 81901557, to Ping Yin)

\section{Availability of data and materials}

The data sets used and/or analysed during the current study are available from the corresponding author on reasonable request.

\section{Ethics approval and consent to participate}

This study was approved by the ethics committee of the Third Affiliated Hospital of Guangzhou Medical University. Each patient has signed an informed consent on obtaining and analyzing their clinical data 
prior to the initiation of IVF/ICSI-ET treatment.

\section{Consent for publication}

Each participant had signed the consent form for the use of her personal data for research use and publication.

\section{Competing interests}

All authors have declared no conflict of interest.

\section{References}

1. Takahashi $\mathrm{N}$, et al. Preclinical validation of the new vitrification device possessing a feature of absorbing excess vitrification solution for the cryopreservation of human embryos. J Obstet Gynaecol Res. 2020;46(2):302-9.

2. Huang $B$, et al. Oxidative damage and antioxidative indicators in $48 \mathrm{~h}$ germinated rice embryos during the vitrification-cryopreservation procedure. Plant Cell Rep. 2018;37(9):1325-42.

3. Van Den Abbeel E, Van Steirteghem A. Zona pellucida damage to human embryos after cryopreservation and the consequences for their blastomere survival and in-vitro viability. Hum Reprod. 2000;15(2):373-8.

4. Rall WF, Fahy GM. Ice-free cryopreservation of mouse embryos at -196 degrees $C$ by vitrification. Nature. 1985;313(6003):573-5.

5. Kasai M. Advances in the cryopreservation of mammalian oocytes and embryos: Development of ultrarapid vitrification. Reprod Med Biol. 2002;1(1):1-9.

6. Son WY, Tan SL. Comparison between slow freezing and vitrification for human embryos. Expert Rev Med Devices. 2009;6(1):1-7.

7. Zegers-Hochschild F, et al. The International Committee for Monitoring Assisted Reproductive Technology (ICMART) and the World Health Organization (WHO) Revised Glossary on ART Terminology, 2009. Hum Reprod. 2009;24(11):2683-7.

8. Loutradi KE, et al. Cryopreservation of human embryos by vitrification or slow freezing: a systematic review and meta-analysis. Fertil Steril. 2008;90(1):186-93.

9. Li Z, et al. Clinical outcomes following cryopreservation of blastocysts by vitrification or slow freezing: a population-based cohort study. Hum Reprod. 2014;29(12):2794-801.

10. Jones HW Jr, Veeck LL, Muasher SJ. Cryopreservation: the problem of evaluation. Hum Reprod. 1995;10(8):2136-8.

11. Keivanloo S, Sudagar M. Cryopreservation of Persian sturgeon (Acipenser persicus) embryos by DMSO-based vitrificant solutions. Theriogenology. 2016;85(5):1013-8.

12. Siebzehnruebl ER, et al. Cryopreservation of human and rabbit oocytes and one-cell embryos: a comparison of DMSO and propanediol. Hum Reprod. 1989;4(3):312-7. 
13. Machtinger R, et al. The effect of prolonged cryopreservation on embryo survival. Gynecol Endocrinol. 2002;16(4):293-8.

14. Testart $\mathrm{J}$, et al. Factors influencing the success rate of human embryo freezing in an in vitro fertilization and embryo transfer program. Fertil Steril. 1987;48(1):107-12.

15. Lavara R, Baselga M, Vicente JS. Does storage time in LN2 influence survival and pregnancy outcome of vitrified rabbit embryos? Theriogenology. 2011;76(4):652-7.

16. Li W, et al. Influence of storage time on vitrified human cleavage-stage embryos froze in open system. Gynecol Endocrinol. 2017;33(2):96-9.

17. Cohen J, et al. Duration of storage of cryopreserved human embryos. J In Vitro Fert Embryo Transf. 1988;5(5):301-3.

18. Mazur P. Freezing of living cells: mechanisms and implications. Am J Physiol. 1984;247(3 Pt 1):C125-42.

19. Martinez-Burgos $M$, et al. Vitrification versus slow freezing of oocytes: effects on morphologic appearance, meiotic spindle configuration, and DNA damage. Fertil Steril. 2011;95(1):374-7.

20. Vanderzwalmen P, et al. [Vitrification and the use of high concentrations of cryoprotectants: is it a justified argument to prefer slow freezing?]. Gynecol Obstet Fertil. 2010;38(9):536-40.

21. AbdelHafez FF, et al. Slow freezing, vitrification and ultra-rapid freezing of human embryos: a systematic review and meta-analysis. Reprod Biomed Online. 2010;20(2):209-22.

22. $\mathrm{Li} \mathrm{J}$, et al. The effect of storage time after vitrification on pregnancy and neonatal outcomes among 24698 patients following the first embryo transfer cycles. Hum Reprod. 2020;35(7):1675-84.

\section{Tables}

Due to technical limitations, table $1,2,3,4$ is only available as a download in the Supplemental Files section.

\section{Supplementary Files}

This is a list of supplementary files associated with this preprint. Click to download.

- table.xlsx 